\title{
Physics of Radiatively Driven Winds by High Angular Resolution Observations (HARO)
}

\author{
Ph. Stee, D. Bonneau, D. Mourard, and F. Vakili \\ Equipe GI2T, Département Fresnel \\ CNRS-UMR 6528 \\ Observatoire de la Côte d'Azur \\ 2130 route de l'Observatoire \\ Caussols \\ 06460 St Vallier de Thiey, France
}

\begin{abstract}
Numerous models have been developed during the last two decades which try to fit a small number of "classical" observables as closely as possible (i.e $\mathrm{H} \alpha$ line profile and continuum energy distribution or polarization data). Nevertheless, little has been done to include High Angular Resolution Observations (HARO) in simulations in spite of the fact that such data can strongly constrain radiative wind models. In the following, we shall review recent results coming from HARO of active $B$ stars and we shall focus on our radiative wind model for active hot stars which integrates these measurements.
\end{abstract}

In order to interpret HARO, we have built a latitude dependent radiative wind model which produces both spectroscopic, photometric, and interferometric synthetic data that have been compared to our interferometric observations. The line force in our model is the same as that used by Friend \& Abbott (1986), but we introduce a varying contribution of thin and thick lines from pole to equator by adopting latitude dependent radiative parameters. We have performed a numerical calculation for parameters characteristic of the Be star $\gamma$ Cas. Our main results are summarized in Table 1 (from Stee 1996). This model indicates that a radiative wind, driven mainly by optically thin lines at the equator, is a likely scenario for $\gamma$ Cas. This is discussed in greater depth in Stee et al. (1995). We have also studied the rotational component of the wind in the envelope of $\gamma$ Cas and we have found that it must be close to Keplerian rotation (Stee, 1996). A global picture of $\gamma$ Cas circumstellar environment is shown in figure 1.

We have carried the first spectrally-resolved observations of $\gamma$ Cas in the He I $\lambda 6678$, and $\mathrm{H} \beta$ emission lines using the GI2T (Stee et al., 1998). We concluded that the $\mathrm{H} \beta$ emitting region must be smaller than 8.5 stellar radius and close to 2.3 stellar radii in He I $\lambda 6678$. This is, for He I $\lambda 6678$, smaller than the nearby continuum extent. These results confirmed the $\gamma$ Cas basic parameters for this star obtained by Stee et al., 1995 (see Table 1). A picture of $\gamma$ Cas as a function of line and wavelengths is shown in figure 2 . 
Table 1. Parameters and results for $\gamma$ Cassiopeiae (from Stee 1996)

\begin{tabular}{|c|c|}
\hline \multicolumn{2}{|l|}{ Parameters } \\
\hline Spectral type & B0.5IVe \\
\hline Effective temperature & $25000 \mathrm{~K}$ \\
\hline Mass & $16 \mathrm{M}_{\odot}$ \\
\hline Radius & $10 \mathrm{R}_{\odot}$ \\
\hline Stellar angular diameter & 0.45 mas \\
\hline Luminosity & $3.510^{4} \mathrm{~L}_{\odot}$ \\
\hline$V \sin i$ & $230 \mathrm{~km} \mathrm{~s}^{-1}$ \\
\hline Inclination angle $i$ & $45^{\circ}$ \\
\hline \multicolumn{2}{|l|}{ Results } \\
\hline Polar terminal velocity & $2016 \mathrm{~km} \mathrm{~s}^{-1}$ \\
\hline Polar mass flux & $1.7 \cdot 10^{-9} \quad \mathrm{M}_{\odot} \mathrm{yr}^{-1} \mathrm{sr}^{-1}$ \\
\hline \multicolumn{2}{|c|}{ Equatorial terminal velocity $200 \mathrm{~km} \mathrm{~s}^{-1}$} \\
\hline Equatorial mass flux & $5.1 \cdot 10^{-8} \quad \mathrm{M}_{\odot} \mathrm{yr}^{-1} \mathrm{sr}^{-1}$ \\
\hline Mass loss rate & $3.2 \cdot 10^{-7} \mathrm{M}_{\odot} \mathrm{yr}^{-1}$ \\
\hline H $\alpha$ major axis & 17 stellar radii \\
\hline $\mathrm{H} \alpha$ oblateness & 0.72 \\
\hline H $\alpha$ extension & 4 mas \\
\hline Mass of the disk & $6.4 \cdot 10^{-8}$ \\
\hline
\end{tabular}

\section{A model for $\gamma$ Cassiopeiae}

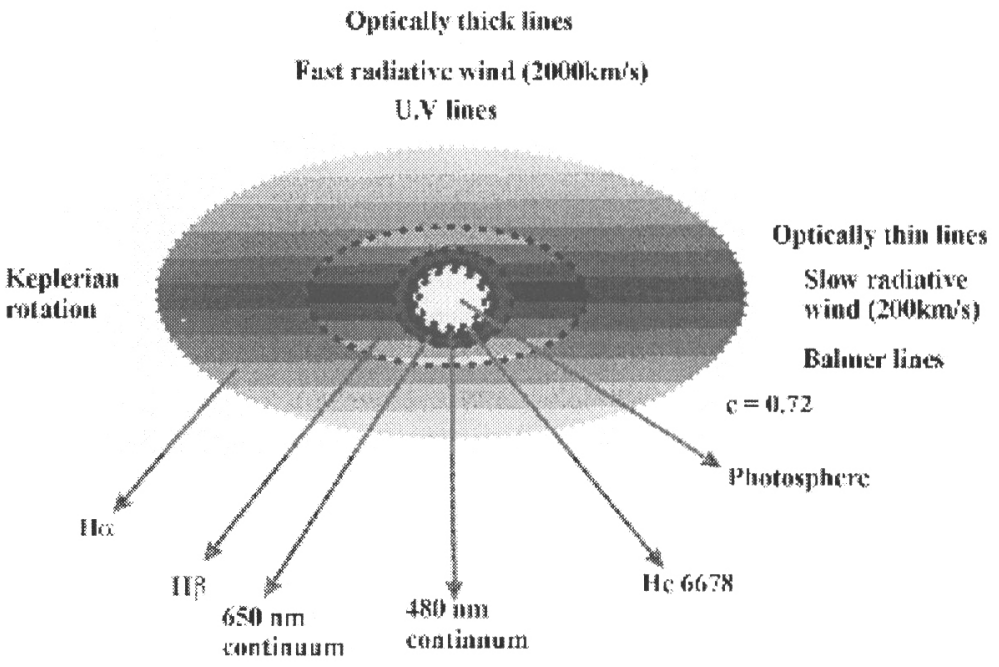

Fig. 1. A picture of $\gamma$ Cas circumstellar environment from a comparison between our radiative wind model and high angular resolution observations. 
Finally our results show that a lemniscat-shaped circumstellar envelope (axisymmetric thin envelopes at the inner edge and thick at the outher rim) is completely inappropriate for $\gamma$ Cas (see for instance hydrostatic disks models proposed by Hanuschik, 1996). Moreover, a theoritical study by Stee (1998) on $\mathrm{B}[\mathrm{e}]$ supergiant disks seems to discard the "wind-compressed disk" (WCD) model proposed by Bjorkman \& Cassinelli (1993) as a possible senario for the envelope of these supergiants.

\section{y Cas at different wavelengths}

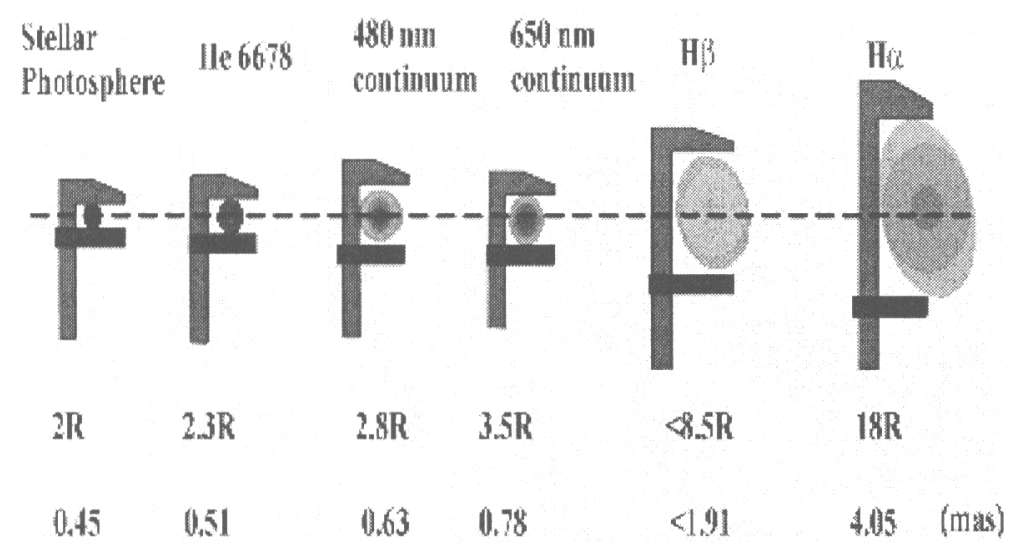

Fig. 2. A schematic view of $\gamma$ Cas as a function of wavelength from our model according to GI2T observations. These envelope wavelength dependent shapes are projections onto the sky plane. The generic "slide-calliper" mimics the N-S orientation of GI2T's baseline.

\section{References}

Bjorkman, J. E., \& Cassinelli, J. P. 1993, ApJ, 409, 429

Friend, D.B., Abbott, D.C. 1986, ApJ, 311,701

Hanuschik, R.W. 1996, A\&A, 308, 170

Stee, Ph., Araújo, F.X., Vakili, F., Mourard, D., Arnold, L., Bonneau, D., Morand, F. and Tallon-Bosc, I. 1995, A\&A, 300, 219

Stee, Ph. 1996, A\&A, 311, 945

Stee, Ph., Vakili, F., Mourard, D., Bonneau, D. 1998, A\&A, 332, 268

Stee, Ph. 1998, A\&A, 336, 980 


\section{Discussion}

T. Eversberg: What is the principle idea to solve the baseline accuracy problem on very large scales like the earth's orbit?

P. Stee: The technique (which is effectively applied on ground-based interferometers: Mark III, PT I at Palomar, etc.) is a nano-metric laser-metrology, which in addition to a "dual feed" possibility (a bright reference star in the field of view) make micro-arcsecond interferometry foreseeable in the next decade.

A. Chalabaev: Your model is in contradiction with the major observational discovery made by the Petrov group, namely that the envelope of $\gamma$ Cas is not centered on the B star, but on an unseen object.

P. Stee: The results presented by the Petrov group in the thesis by Sanchez are not really convincing; it is not possible to explain these observations with any model, even if $\gamma$ Cas is not centered on the B star. Concerning the new results you mentioned: I am not aware of them, as they are not yet published in any refereed paper. However, having a photocenter displacement with respect to the star does not mean that the matter is not centered on the star.

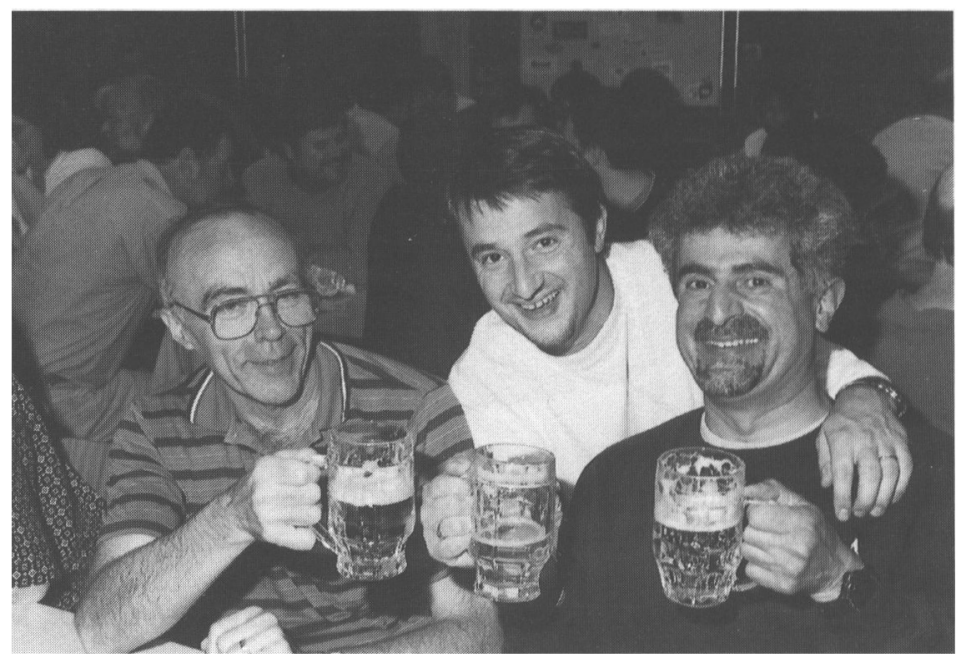

Michael Marlborough, Philippe Stee and Farrokh Vakili 